\title{
LACRIMAL SURGERY IN PATIENTS WITH CONGENITAL CRANIAL OR FACIAL ANOMALIES
}

\author{
CELIA HICKS, JOHN PITTS and GEOFFREY E. ROSE \\ London
}

\begin{abstract}
SUMMARY
A series of 26 patients with lacrimal dysfunction due to congenital cranial or facial anomalies are reviewed. The underlying anatomical abnormalities responsible for symptoms and their influence on surgical management and outcome are discussed and illustrated with representative cases. It is shown that by adaptation of standard surgical techniques a high success rate can be achieved.
\end{abstract}

The eliminatory component of the lacrimal system begins to develop early in embryogenesis and genetic or exogenous factors which influence development at this stage are likely to result in lacrimal disorders.'

At about day 45 of embryogenesis, a cord of surface ectoderm becomes buried ${ }^{2}$ when the lateral nasal process and the maxillary process on each side meet, and two solid epithelial cords derived from it extend into the developing lids; these reach the lid margins by the $35 \mathrm{~mm}$ stage and gradually canalise from their central portions towards the lid margins. The nasolacrimal duct itself begins to canalise during the fourth month in a patchy fashion, the nasal end opening last, and the system may not be fully functional at birth.

Malunion of the maxillary and nasal processes due to chromosomal abnormalities or intrauterine factors, such as amniotic bands, can result in a variety of clefting defects which are often associated with other anomalies. Any part of the lacrimal drainage system may be involved and may be open to the surface, partially constricted or totally absent. Congenital fistulae of the lacrimal sac can arise due to failed obliteration of the optic end of the nasooptic groove; fistulae can also develop secondarily to chronic dacryocystitis resulting from inadequate sac drainage of any cause. Canalisation of any part of the system may fail. Deformities of facial development resulting in malposition of the orbits, hypertelorism or abnormal lid attachments will also give rise to disturbance of lacrimal function.

Correspondence to: Mr G. E. Rose, Adnexal Service, Moorfields Eye Hospital, City Road, London EC1V 2PD, UK.
The 26 patients in this series with congenital cranial or facial abnormalities all presented with epiphora and 10 with mucocoeles. In many cases there were multiple abnormalities contributing to symptoms, but in each case the main cause of failure of drainage could be localised to one part of the system: lid malposition or malfunction; clefting, stenosis or agenesis of the canaliculus, sac or duct; or midline anomalies such as choanal atresia or telecanthus. Factors which influence management and outcome in these groups of patients are discussed.

\section{PATIENTS AND METHODS}

Patients with lacrimal disorders associated with cranial or facial malformations were identified from clinic and operating records and their notes, clinical photographs and radiographs were reviewed.

\section{RESULTS}

A heterogeneous group of 26 patients, fifteen female and eleven male, who presented between 1980 and 1993 was identified (Table I).

The clinical data from the patients may be grouped into those with symptoms due primarily to lid abnormalities (Table II), canalicular abnormalities (Table III), lacrimal sac (Table IV), duct agenesis associated with clefting (Table V), duct stenosis associated with clefting (Table VI) and midline abormalities, including absence of external nares (Table VII).

Table I. Disorders presented by the patients

\begin{tabular}{lc}
\hline Disorder & No. of patients \\
\hline Clefting with associated anomalies & 6 \\
Simple facial clefts or amniotic bands & 4 \\
Ectodermal dysplasia & 4 \\
Telecanthus and associated anomalies & 3 \\
Rubenstein-Taybi syndrome & 2 \\
Fraser's syndrome & 2 \\
Treacher-Collins syndrome & 1 \\
Miller's syndrome & 1 \\
Craniometaphyseal dysplasia & 1 \\
Goldenhar syndrome & 1 \\
Saethre-Chotzen syndrome & 1 \\
\hline
\end{tabular}


Table II. Epiphora associated with lid abnormalities

\begin{tabular}{|c|c|c|c|c|c|c|c|}
\hline Case & $\begin{array}{l}\text { Sex and } \\
\text { age }\end{array}$ & Symptoms & Ocular findings & Diagnosis & Other features & $\begin{array}{c}\text { Treatment } \\
\text { (age in years) }\end{array}$ & Outcome \\
\hline 1 & 6 months & $\begin{array}{l}\text { Epiphora; multiple } \\
\text { bilateral } \\
\text { dacryocystitis }\end{array}$ & $\begin{array}{l}\text { Medially displaced } \\
\text { puncta }\end{array}$ & $\begin{array}{l}\text { Rubenstein-Taybi } \\
\text { syndrome }\end{array}$ & $\begin{array}{l}\text { Mental handicap; } \\
\text { broad stumpy fingers }\end{array}$ & $\begin{array}{l}\text { L DCR (5), failed due } \\
\text { to adhesions. L redo- } \\
\text { DCR (6) with tubes }\end{array}$ & $\begin{array}{l}\text { Discharged } \\
\text { symptom- } \\
\text { free }\end{array}$ \\
\hline 2 & $\begin{array}{c}\mathrm{F} \\
5 \mathrm{yr}\end{array}$ & R epiphora & $\begin{array}{l}\text { Abnormal position } \\
\text { of medial canthal } \\
\text { tendon; strabismus }\end{array}$ & $\begin{array}{l}\text { Rubenstein-Taybi } \\
\text { syndrome }\end{array}$ & Polydactyly & $\begin{array}{l}\text { Probed }(5,6 \& 8 \\
\text { months). R DCR (1), } \\
\text { with medial } \\
\text { canthoplasty. Large } \\
\text { sac and very thick } \\
\text { bone; histology } \\
\text { revealed reactive new } \\
\text { bone formation }\end{array}$ & $\begin{array}{l}\text { Discharged } \\
\text { symptom- } \\
\text { free }\end{array}$ \\
\hline 3 & $\begin{array}{c}\mathrm{M} \\
1 \mathrm{yr}\end{array}$ & Bilateral epiphora & $\begin{array}{l}\text { Absent lower puncta; } \\
\text { abnormal lashes }\end{array}$ & $\begin{array}{l}\text { Treacher-Collins } \\
\text { syndrome }\end{array}$ & & $\begin{array}{l}\text { EUA; planned DCR } \\
\text { cancelled as } \\
\text { asymptomatic after } 1 \\
\text { year review }\end{array}$ & Discharged \\
\hline 5 & $\begin{array}{c}\mathrm{M} \\
15 \mathrm{yr}\end{array}$ & L epiphora & $\begin{array}{l}\text { L upper lid } \\
\text { coloboma: } \\
\text { telecanthus; } \\
\text { synophthalmos; } \\
\text { ankyloblepharon; } \\
\text { strabismus }\end{array}$ & Fraser's syndrome & $\begin{array}{l}\text { Abnormal ear; floppy } \\
\text { larynx; single } \\
\text { abnormal kidney; } \\
\text { hypospadias }\end{array}$ & $\begin{array}{l}\text { EUA ( } 1 \text { yr), with } \\
\text { symblepharon and } \\
\text { band division with } \\
\text { conjunctival bridge } \\
\text { flap from lower } \\
\text { fornix; lateral } \\
\text { cantholysis }\end{array}$ & $\begin{array}{l}\text { Discharged } \\
\text { with fewer } \\
\text { symptoms }\end{array}$ \\
\hline 6 & $\begin{array}{c}\text { M } \\
7 \text { days }\end{array}$ & L epiphora & $\begin{array}{l}\text { L upper lid coloboma } \\
\text { dermoid }\end{array}$ & Goldenhar syndrome & & $\begin{array}{l}\text { Probing ( } 10 \text { days) at } \\
\text { time of coloboma } \\
\text { closure }\end{array}$ & $\begin{array}{l}\text { Discharged } \\
\text { symptom- } \\
\text { free }\end{array}$ \\
\hline
\end{tabular}

M, male; F, female; R, right; L, left; DCR, dacryocystorhinostomy; EUA, examination under anaesthesia; NLD. nasolacrimal duct.

Of the 26 patients reviewed, 2 patients were cured by syringe and probing alone and 1 patient's symptoms ameliorated spontaneously whilst awaiting dacryocystorhinostomy. Eight patients required lid surgery, 6 of these in addition to lacrimal drainage surgery, and 11 patients had unilateral and 8 bilateral dacrystorhinostomy; silicon intubation was used as a primary procedure in 4 patients unilaterally and 2 patients bilaterally. Dacryocystorhinostomy failed in 2 patients, 1 due to intranasal adhesions and 1 due to lack of external nares, that subsequently required ear, nose and throat surgery; in both cases repeat dacryocystorhinostomy with intubation was successful.

The final outcome was successful in 24 patients: 21 have been discharged symptom-free after a minimum follow-up of 3 months and 2 are awaiting surgery to the second side; 1 patient (case 12) failed to attend for removal of tubes. One patient (case 7) remained symptomatic in spite of bilaterally patent systems on the dacryocystogram and 1 patient with ectodermal dysplasia (case 9) subsequently developed a dry eye.

\section{CASE REPORTS}

\section{Case 4: Miller's Syndrome; Lid Abnormalities}

A 5-year-old girl was referred after two unsuccessful probings and intubation for bilateral epiphora. She exhibited many of the typical features of Miller's syndrome, an autosomal recessive condition characterised by malar and mandibular hypoplasia, clefting, hand and toe deformities, abnormal ears and absent tooth enamel. She had bilateral seventh nerve palsy, with lower lid ectropion, in addition to absent upper puncta and distal duct stenosis on dacryocystography. On the right side there was a fistula between the lower canaliculus and the lower lid.

Symptoms resolved completely following lateral canthal tightening to improve lid position and augment the lacrimal pump (Figs. 1,2) combined with dacryocystorhinostomy to reduce distal resistance.

\section{Case 8: Ectrodactyly-Ectodermal Dysplasia- Clefting (EEC); Canalicular Abnormality}

This boy presented at 4 years of age with bilateral 
Table III. Epiphora associated with canalicular abnormality

\begin{tabular}{|c|c|c|c|c|c|c|c|}
\hline Case & $\begin{array}{l}\text { Sex and } \\
\text { age }\end{array}$ & Symptoms & Ocular findings & Diagnosis & Other features & $\begin{array}{c}\text { Treatment } \\
\text { (age in years) }\end{array}$ & Outcome \\
\hline 7 & $\begin{array}{c}\mathrm{M} \\
4 \mathrm{yr}\end{array}$ & Bilateral epiphora & $\begin{array}{l}\text { Absent left lower } \\
\text { punctum }\end{array}$ & EEC syndrome & $\begin{array}{l}\text { Absent tooth enamel; } \\
\text { deaf; hypohydrosis; } \\
\text { submucous cleft } \\
\text { palate; syndactyly }\end{array}$ & $\begin{array}{l}\text { Probing (4); no } \\
\text { communication } \\
\text { between upper } \\
\text { canaliculus and sac, } \\
\text { or between sac and } \\
\text { nose on either side. } \\
\text { Bilateral; DCR (5) } \\
\text { prominent middle } \\
\text { turbinate fractured }\end{array}$ & $\begin{array}{l}\text { Discharged } \\
\text { with } \\
\text { symptoms } \\
\text { despite } \\
\text { patent } \\
\text { systems }\end{array}$ \\
\hline 8 & $\begin{array}{c}\mathrm{M} \\
4 \mathrm{yr}\end{array}$ & $\begin{array}{l}\text { Bilateral epiphora; } \\
\text { chronic } \\
\text { conjunctivitis; } \\
\text { photophobia }\end{array}$ & $\begin{array}{l}\text { Absence of lower } \\
\text { puncta; } \\
\text { blepharophimosis; } \\
\text { absent meibomian } \\
\text { glands, entropion and } \\
\text { conjunctival } \\
\text { squamous metaplasia }\end{array}$ & EEC syndrome & $\begin{array}{l}\text { Bilateral cleft lip and } \\
\text { palate; severe lobster } \\
\text { claw hands and feet; } \\
\text { sparse hair; low-set } \\
\text { ears }\end{array}$ & $\begin{array}{l}\text { R DCR (4), L DCR } \\
\text { (5); in both cases a } \\
\text { pit in the lower lid } \\
\text { was incised for tube } \\
\text { placement }\end{array}$ & $\begin{array}{l}\text { Discharged } \\
\text { symptom- } \\
\text { free }\end{array}$ \\
\hline 9 & $\begin{array}{c}F \\
8 \mathrm{yr}\end{array}$ & $\begin{array}{l}\mathrm{R} \text { epiphora and } \\
\text { mucocoele }\end{array}$ & $\begin{array}{l}\text { Dry eyes with pannus } \\
\text { and corneal scars; } \\
\text { keratin plaques; } \\
\text { absent upper puncta, } \\
\text { lower keratinised; } R \\
\text { lower canaliculus } \\
\text { patent to } 2 \mathrm{~mm}\end{array}$ & Ectodermal dysplasia & $\begin{array}{l}\text { Abnormal fingers, } \\
\text { toes and teeth }\end{array}$ & R DCR (10) & $\begin{array}{l}\text { No further } \\
\text { treatment } \\
\text { because of } \\
\text { keratitis } \\
\text { sicca }\end{array}$ \\
\hline 10 & $\begin{array}{c}\mathrm{F} \\
2 \text { weeks }\end{array}$ & Bilateral epiphora & $\begin{array}{l}\text { Bilateral occlusion } \\
\text { of upper and lower } \\
\text { puncta by membranes }\end{array}$ & $\begin{array}{l}\text { Clefting with multiple } \\
\text { defects }\end{array}$ & $\begin{array}{l}\text { Bilateral cleft palate; } \\
\text { malar hypoplasia; } \\
\text { abnormal ear; } \\
\text { deafness; } \\
\text { polydactyly; dry skin }\end{array}$ & $\begin{array}{l}\text { L DCR ( } 1) \text {, R DCR } \\
\text { (2); L canaliculus } \\
\text { normal beyond } \\
\text { punctum; abnormal } \\
\text { lower canaliculus on } \\
\text { R }\end{array}$ & $\begin{array}{l}\text { Discharged } \\
\text { symptom- } \\
\text { free }\end{array}$ \\
\hline
\end{tabular}

Table IV. Epiphora associated with lacrimal sac abnormality

\begin{tabular}{|c|c|c|c|c|c|c|c|}
\hline Case & $\begin{array}{l}\text { Sex and } \\
\text { age }\end{array}$ & Symptoms & Ocular findings & Diagnosis & Other features & $\begin{array}{c}\text { Treatment } \\
\text { (age in years) }\end{array}$ & Outcome \\
\hline 11 & $\underset{1 \mathrm{yr}}{\mathrm{F}}$ & $\begin{array}{l}\mathrm{L} \text { recurrent } \\
\text { dacryocystitis }\end{array}$ & $\begin{array}{l}\text { Congenital absence } \\
\text { of L upper lid; large } \\
\text { L mucocoele } \\
\text { discharging via } 2 \\
\text { acquired fistulae; } \\
\text { strabismus }\end{array}$ & $\begin{array}{l}\text { Clefting secondary to } \\
\text { amniotic bands }\end{array}$ & $\begin{array}{l}\text { Occipital defect } \\
\text { requiring V-P shunt; } \\
\text { hand abnormalities }\end{array}$ & $\begin{array}{l}\text { Tarsorrhaphy and } \\
\text { plastic surgery to the } \\
\text { upper lid, scalp } \\
\text { defect closure by } \\
\text { Z-plasty, and V-P } \\
\text { shunt were all } \\
\text { performed in infancy; } \\
\text { DCG showed band } \\
\text { across lacrimal sac. } \\
\text { Abnormal position of } \\
\text { caruncle and puncta. } \\
\text { L DCR (1) }\end{array}$ & $\begin{array}{l}\text { Discharged } \\
\text { symptom- } \\
\text { free }\end{array}$ \\
\hline 12 & $\begin{array}{c}\mathrm{M} \\
26 \mathrm{yr}\end{array}$ & Bilateral epiphora & $\begin{array}{l}\text { Telecanthus; anti- } \\
\text { mongoloid slant }\end{array}$ & Craniofacial anomaly & $\begin{array}{l}\text { Abnormal face and } \\
\text { ears }\end{array}$ & $\begin{array}{l}\text { L DCR (27); } \\
\text { R CDCR ( } 28) \text {, when } \\
\text { abnormal bone in } \\
\text { fossa and absent R } \\
\text { lacrimal sac found }\end{array}$ & $\begin{array}{l}\text { Symptom- } \\
\text { free post- } \\
\text { operatively }\end{array}$ \\
\hline
\end{tabular}

V-P, ventriculo-peritoneal.

Table V. Epiphora associated with duct agenesis and clefting

\begin{tabular}{|c|c|c|c|c|c|c|c|}
\hline Case & $\begin{array}{l}\text { Sex and } \\
\text { age }\end{array}$ & Symptoms & Ocular findings & Diagnosis & Other features & $\begin{array}{c}\text { Treatment } \\
\text { (age in years) }\end{array}$ & Outcome \\
\hline 13 & $\underset{3 \mathrm{yr}}{\mathrm{F}}$ & Bilateral epiphora & $\begin{array}{l}\text { Absent L NLD } \\
\text { (DCG) }\end{array}$ & EEC syndrome & $\begin{array}{l}\text { Bilateral cleft palate } \\
\text { and mandibular } \\
\text { hypoplasia; abnormal } \\
\text { hands and feet }\end{array}$ & $\begin{array}{l}\mathrm{R} \text { DCR }(5) ; \mathrm{L} \mathrm{DCR} \\
\text { and middle turbinate } \\
\text { fracture (5) }\end{array}$ & $\begin{array}{l}\text { Discharged } \\
\text { symptom- } \\
\text { free }\end{array}$ \\
\hline 14 & $\begin{array}{c}\mathrm{M} \\
1 \mathrm{yr}\end{array}$ & R epiphora & Congenital sac fistula & $\begin{array}{l}\text { Nasolacrimal duct } \\
\text { agenesis due to } \\
\text { clefting }\end{array}$ & $\mathrm{R}$ cleft lip and palate & $\begin{array}{l}\text { R DCR (1); fistula } \\
\text { curretted }\end{array}$ & $\begin{array}{l}\text { Discharged } \\
\text { symptom- } \\
\text { free }\end{array}$ \\
\hline 15 & $\begin{array}{c}\mathrm{F} \\
6 \mathrm{yr}\end{array}$ & $\begin{array}{l}\mathrm{R} \text { epiphora and } \\
\text { mucocoele }\end{array}$ & $\begin{array}{l}\mathrm{L} \text { amblyopia, absent } \\
\text { lower punctum and } \\
\text { lower lid entropion; } \\
\mathrm{R} \text { congenital sac } \\
\text { fistula }\end{array}$ & $\begin{array}{l}\mathrm{R} \text { nasolacrimal duct } \\
\text { agenesis due to } \\
\text { clefting }\end{array}$ & $\begin{array}{l}\text { Abnormal } L \text { side face } \\
\text { and eye; bilateral cleft } \\
\text { lip and palate }\end{array}$ & R DCR (6) & $\begin{array}{l}\text { Discharged } \\
\text { symptom- } \\
\text { free }\end{array}$ \\
\hline
\end{tabular}


Table VI. Epiphora associated with duct abnormalities and clefting

\begin{tabular}{|c|c|c|c|c|c|c|c|}
\hline Case & $\begin{array}{l}\text { Sex and } \\
\text { age }\end{array}$ & Symptoms & Ocular findings & Diagnosis & Other features & $\begin{array}{c}\text { Treatment } \\
\text { (age in years) }\end{array}$ & Outcome \\
\hline 16 & $\begin{array}{c}\mathrm{F} \\
17 \mathrm{yr}\end{array}$ & R epiphora & $\begin{array}{l}\mathrm{R} \text { nasolacrimal duct } \\
\text { open to surface; } \mathrm{R} \\
\text { ectropion due to } \\
\text { tethering band }\end{array}$ & Clefting & $\mathrm{R}$ cleft lip and palate & $\begin{array}{l}\text { Cleft closed in } \\
\text { infancy: excision } \\
\text { band, posterofixation } \\
\text { of lid (17), and R } \\
\text { DCR (18) }\end{array}$ & $\begin{array}{l}\text { Discharged } \\
\text { symptom- } \\
\text { free }\end{array}$ \\
\hline 17 & $\begin{array}{c}\mathrm{F} \\
1 \mathrm{yr}\end{array}$ & $\mathrm{R}$ epiphora & & $\begin{array}{l}\text { Clefting with } \\
\text { multiple } \\
\text { abnormalities }\end{array}$ & $\begin{array}{l}\mathrm{R} \text { cleft palate and lip; } \\
\text { broad nasal bridge; } \\
\text { large tongue: } \\
\text { hypothyroid }\end{array}$ & $\begin{array}{l}\text { DCG and probing } \\
\text { (4); system patent }\end{array}$ & $\begin{array}{l}\text { Discharged } \\
\text { symptom- } \\
\text { free }\end{array}$ \\
\hline 18 & $\begin{array}{c}\mathrm{F} \\
3 \mathrm{yr}\end{array}$ & $\begin{array}{l}\mathrm{R} \text { epiphora and } \\
\text { mucocoele }\end{array}$ & $\begin{array}{l}\text { Blind R eye with } \\
\text { coloboma: aperture } \\
\text { slanted and displaced } \\
\text { temporally: ectropion; } \\
\text { absent puncta; fistula } \\
\text { between lacrimal } \\
\text { gland and skin }\end{array}$ & $\begin{array}{l}\text { Clefting with } \\
\text { holoprosencephaly }\end{array}$ & $\begin{array}{l}\text { Cleft lip and palate; } \\
\text { duplex kidney: } \\
\text { epilepsy }\end{array}$ & $\begin{array}{l}\text { DCG and probing } \\
\text { (4): repeat probing } \\
\text { after symptom } \\
\text { recurrence }(10) \text { : } \mathrm{L} \\
\text { system normal; } \mathrm{R} \\
\text { stenotic duct with } \\
\text { dilated lower third but } \\
\text { free flow to nose }\end{array}$ & $\begin{array}{l}\text { Discharged } \\
\text { symptom- } \\
\text { free }\end{array}$ \\
\hline 19 & $\begin{array}{c}\mathrm{M} \\
3 \mathrm{yr}\end{array}$ & $\begin{array}{l}\text { Bilateral epiphora } \\
\text { and mucocoeles }\end{array}$ & $\begin{array}{l}\text { Megalocornea: } \\
\text { telecanthus: } \\
\text { exotropia }\end{array}$ & $\begin{array}{l}\text { Clefting with } \\
\text { multiple } \\
\text { abnormalities }\end{array}$ & $\begin{array}{l}\text { High and cleft palate; } \\
\text { syndactyly }\end{array}$ & $\begin{array}{l}\text { L DCR (3); R DCR } \\
\text { (3); hypertelorism } \\
\text { correction (8) }\end{array}$ & $\begin{array}{l}\text { Discharged } \\
\text { symptom- } \\
\text { free }\end{array}$ \\
\hline 20 & $\begin{array}{c}\mathrm{M} \\
9 \mathrm{yr}\end{array}$ & Bilateral epiphora & & $\begin{array}{l}\text { Clefting with } \\
\text { multiple } \\
\text { abnormalities }\end{array}$ & $\begin{array}{l}\text { Multiple facial } \\
\text { abnormalities; cleft } \\
\text { palate; spatulate L } \\
\text { hand }\end{array}$ & $\begin{array}{l}\text { R DCR (9); L DCR } \\
\text { (10) }\end{array}$ & $\begin{array}{l}\text { Discharged } \\
\text { symptom- } \\
\text { free }\end{array}$ \\
\hline
\end{tabular}

Table VII. Epiphora associated with ductal and midline or nasal abnormalities

\begin{tabular}{|c|c|c|c|c|c|c|c|}
\hline Case & $\begin{array}{l}\text { Sex and } \\
\text { age }\end{array}$ & Symptoms & Ocular features & Diagnosis & Other features & $\begin{array}{c}\text { Treatment } \\
\text { (age in years) }\end{array}$ & Outcome \\
\hline 21 & $\underset{1 \mathrm{yr}}{\mathrm{F}}$ & $\begin{array}{l}\text { Bilateral epiphora } \\
\text { and mucocoeles }\end{array}$ & $\begin{array}{l}\mathrm{R} \text { coloboma; } \mathrm{L} \\
\text { phthisis }\end{array}$ & $\begin{array}{l}\text { Midline cleft with } \\
\text { multiple facial } \\
\text { anomalies }\end{array}$ & $\begin{array}{l}\text { Midline nasal defect: } \\
\text { choanal atresia }\end{array}$ & $\begin{array}{l}\text { L DCR ( } 1 \text { ); found } \\
\text { pus-filled nasal cavity } \\
\text { with neither anterior } \\
\text { nor posterior nares. } \\
\text { ENT surgery } \\
\text { performed to open } \\
\text { nares. R DCR }\left(1^{1} / 2\right) \text { : R } \\
\text { redo-DCR }(7)\end{array}$ & $\begin{array}{l}\text { Discharged } \\
\text { symptom- } \\
\text { free age } 7 \\
\text { yr }\end{array}$ \\
\hline 22 & $\begin{array}{c}F \\
24 \mathrm{yr}\end{array}$ & $\begin{array}{l}\text { Bilateral epiphora } \\
\text { and mucocoeles }\end{array}$ & $\begin{array}{l}\text { Telecanthus; bilateral } \\
\text { ptosis }\end{array}$ & Craniofacial anomaly & Large ears and nose & $\begin{array}{l}\text { L DCR (25); very } \\
\text { long medial canthal } \\
\text { tendon; bone } 10 \mathrm{~mm} \\
\text { thick }\end{array}$ & $\begin{array}{l}\text { L } \\
\text { symptom- } \\
\text { free: } \\
\text { awaiting } \\
\text { surgery to } \\
\text { R side }\end{array}$ \\
\hline 23 & $\begin{array}{c}\mathrm{M} \\
36 \mathrm{yr}\end{array}$ & $\begin{array}{l}\text { L epiphora and } \\
\text { mucocoele }\end{array}$ & $\begin{array}{l}\text { Hypertelorism; } \\
\text { vascularised cornea; } \\
\text { defective upper } \\
\text { medial orbital rim }\end{array}$ & $\begin{array}{l}\text { Severe midline } \\
\text { anomaly }\end{array}$ & $\begin{array}{l}\text { Mental retardation; } \\
\text { absent L airway }\end{array}$ & $\begin{array}{l}\mathrm{L} \mathrm{DCR}(36) \text { across } \\
\text { midline to right nasal } \\
\text { space }\end{array}$ & $\begin{array}{l}\text { Discharged } \\
\text { symptom- } \\
\text { free }\end{array}$ \\
\hline 24 & $\begin{array}{c}\mathrm{F} \\
7 \mathrm{yr}\end{array}$ & Bilateral epiphora & $\begin{array}{l}\text { Bilateral corneal } \\
\text { scars, nystagmus and } \\
\text { blind L eye: } \\
\text { hypertelorism, ptosis } \\
\text { and trichiasis }\end{array}$ & Fraser’s syndrome & $\begin{array}{l}\text { Developmental delay; } \\
\text { bilateral external } \\
\text { auditory canal atresia: } \\
\text { cryptophthalmos }\end{array}$ & $\begin{array}{l}\text { Midline facial } \\
\text { reduction in infancy; } \\
\text { L DCR and lateral } \\
\text { canthoplasty ( } 7 \text { ); full- } \\
\text { thickness skin graft } \\
\text { to L lower lid and } \\
\text { retractor release (8) }\end{array}$ & $\begin{array}{l}\text { Cosmesis } \\
\text { and } \\
\text { drainage } \\
\text { much } \\
\text { improved } \\
\text { after DCR; } \\
\text { reduced } \\
\text { lagophthal- } \\
\text { mos and } \\
\text { soreness } \\
\text { after lid } \\
\text { surgery. } \\
\text { Awaiting } \\
\text { surgery to } \\
\text { R side }\end{array}$ \\
\hline 25 & $\begin{array}{c}\mathrm{F} \\
2 \mathrm{yr}\end{array}$ & $\begin{array}{l}\text { L epiphora; } \\
\text { mucocoele }\end{array}$ & Telecanthus & $\begin{array}{l}\text { Craniometaphyseal } \\
\text { dysplasia }\end{array}$ & $\begin{array}{l}\text { Bony dysplasia; } \\
\text { choanal stenosis; L } \\
\text { facial palsy }\end{array}$ & $\begin{array}{l}\text { L DCR (2); L redo- } \\
\text { DCR (4) }\end{array}$ & $\begin{array}{l}\text { Discharged } \\
\text { symptom- } \\
\text { free }\end{array}$ \\
\hline 26 & $\begin{array}{c}\mathrm{M} \\
25 \mathrm{yr}\end{array}$ & $\begin{array}{l}\text { Bilateral epiphora } \\
\text { and mucocoele }\end{array}$ & $\begin{array}{l}\text { Ptosis, telecanthus, } \\
\text { high myopia; L upper } \\
\text { punctum absent }\end{array}$ & $\begin{array}{l}\text { Saethre-Chotzen } \\
\text { syndrome }\end{array}$ & Low-set ears & $\begin{array}{l}\text { L DCR (25); R DCR } \\
\text { (26); bilateral thick } \\
\text { bone noted }\end{array}$ & $\begin{array}{l}\text { Discharged } \\
\text { symptom- } \\
\text { free }\end{array}$ \\
\hline
\end{tabular}




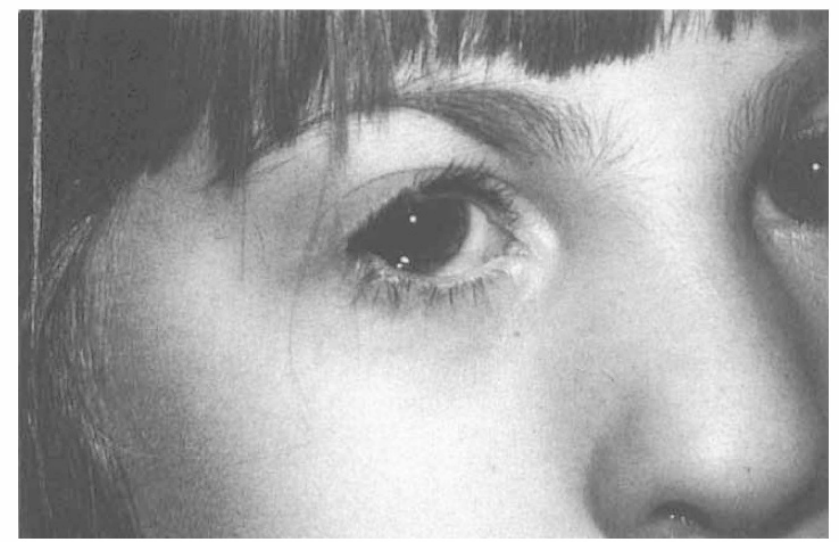

Fig. 1. Case 4. Lateral canthal dystocia and fistula between lower canaliculus and skin.

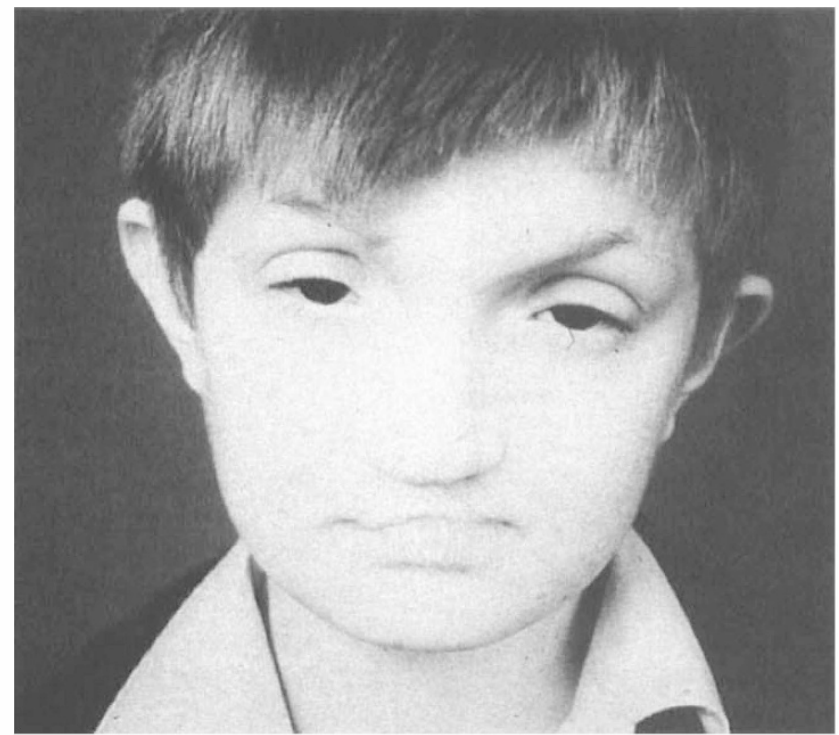

Fig. 3. Case 8. EEC syndrome: facial appearance.

epiphora and chronic conjunctivitis. Both lower puncta were absent, although lower lid entropion and squamous metaplasia of the lid margins contributed to his symptoms. Other deformities included clefting and 'lobster claw' hands and feet (Figs. 3, 4). Dacryocystography confirmed the absence of inferior canaliculi bilaterally and also revealed duct stenosis, with bilateral mucocoele.

Bilateral dacryocystorhinostomies were performed and on each side a pit in the lower lid was incised for placement of silicone tubes. He was discharged symptom-free.

\section{Case 11: Amniotic Banding; Sac Involved in Cleft}

This female child was born with severe clefting defects secondary to amniotic banding; a large occipital defect required ventriculo-peritoneal shunting and closure by Z-plasty soon after birth. Banding had also caused hand abnormalities with finger amputation (Fig. 5) and congenital absence of most of the left upper lid (Fig. 6). Tarsorrhaphy followed by plastic surgery to the left upper lid was performed early in infancy and the cornea remained in good condition.

She developed a large left mucocoele and recurrent

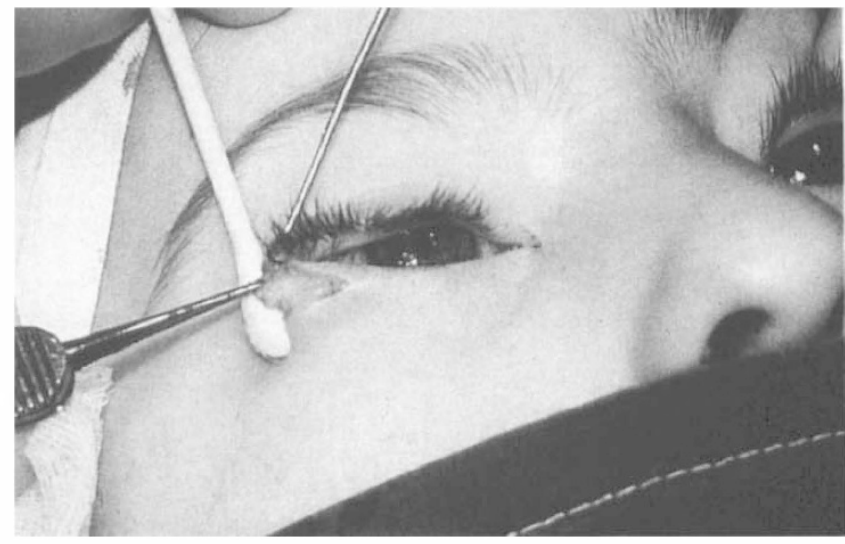

Fig. 2. Case 4. Lateral canthal tightening.

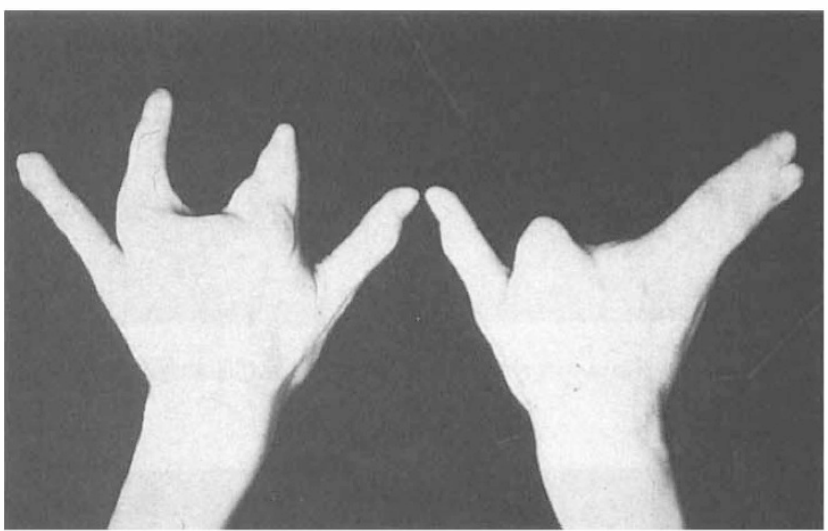

Fig. 4. Case 8. EEC syndrome: hands.

dacryocystitis and at the age of 1 year underwent left dacryocystorhinostomy. The left caruncle was laterally displaced and found to have a punctum on either side of it on the lower lid. The right system was entirely normal. The child was discharged free of symptoms.

\section{Case 16: Clefting; Associated with Duct Stenosis}

This female patient had severe right facial clefting affected the lip and palate and the nasolacrimal duct was open to the surface throughout its length (Fig. 7). There was a band of skin causing right lower lid ectropion (Fig. 7). Initial treatment in infancy involved closure of the cleft (Fig. 8) and in early adulthood excision of the band of skin was performed, with postero-fixation of the lid. After right dacryocystorhinostomy (Fig. 9) the patient was discharged with good cosmesis and free from epiphora.

\section{Case 14: Clefting; Associated with Duct Agenesis}

This male child was born with a right-sided cleft lip and palate, which was repaired in infancy. He had right epiphora and dacryocystitis soon after birth and, within a few 


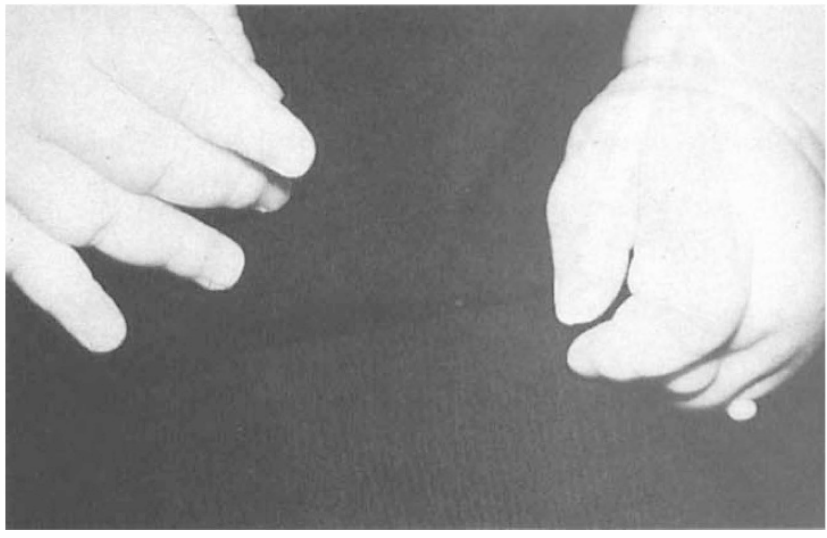

Fig. 5. Case 11. Finger abnormalities due to banding.

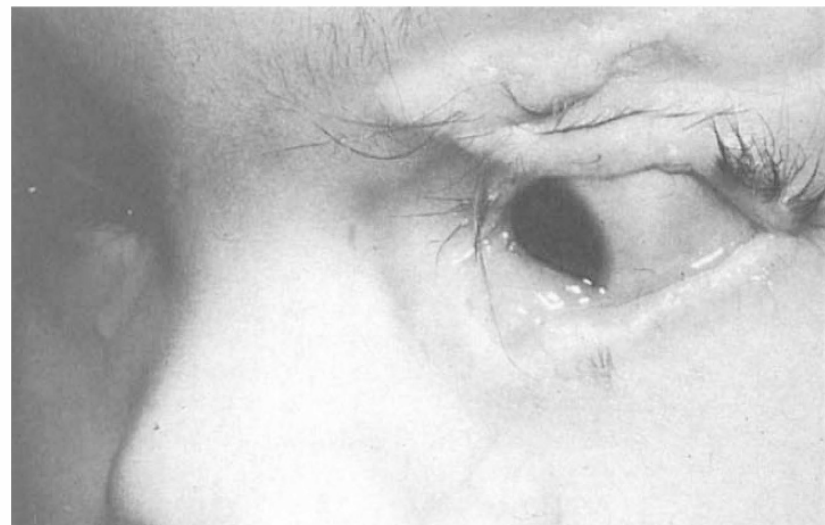

Fig. 6. Case 11. Lid abnormalities due to banding.

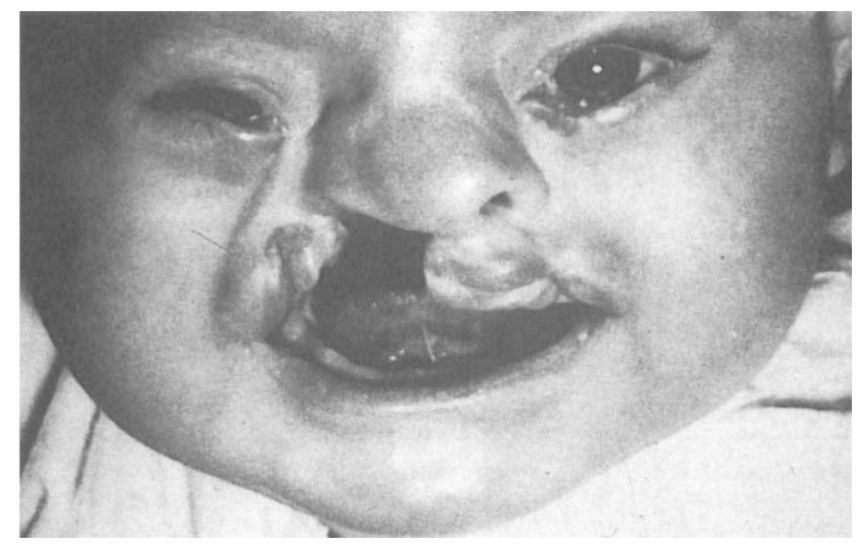

Fig. 7. Case 16. Nasolacrimal duct open to surface.

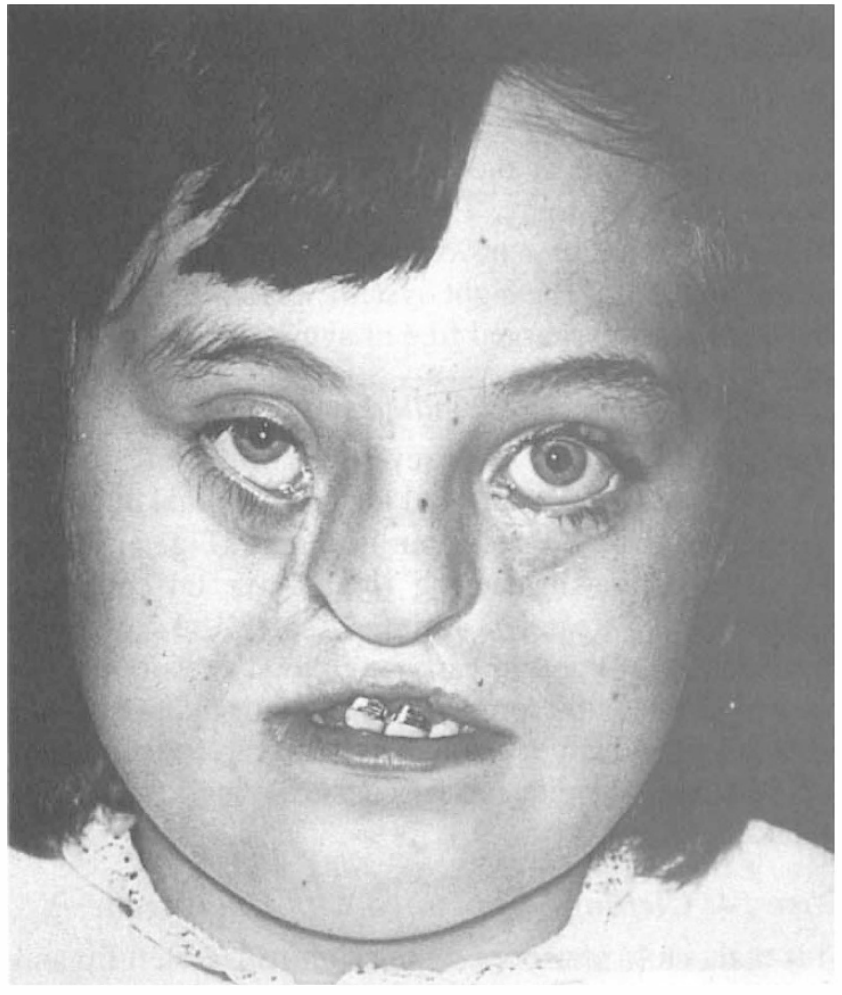

Fig. 8. Case 16. After closure of cleft.

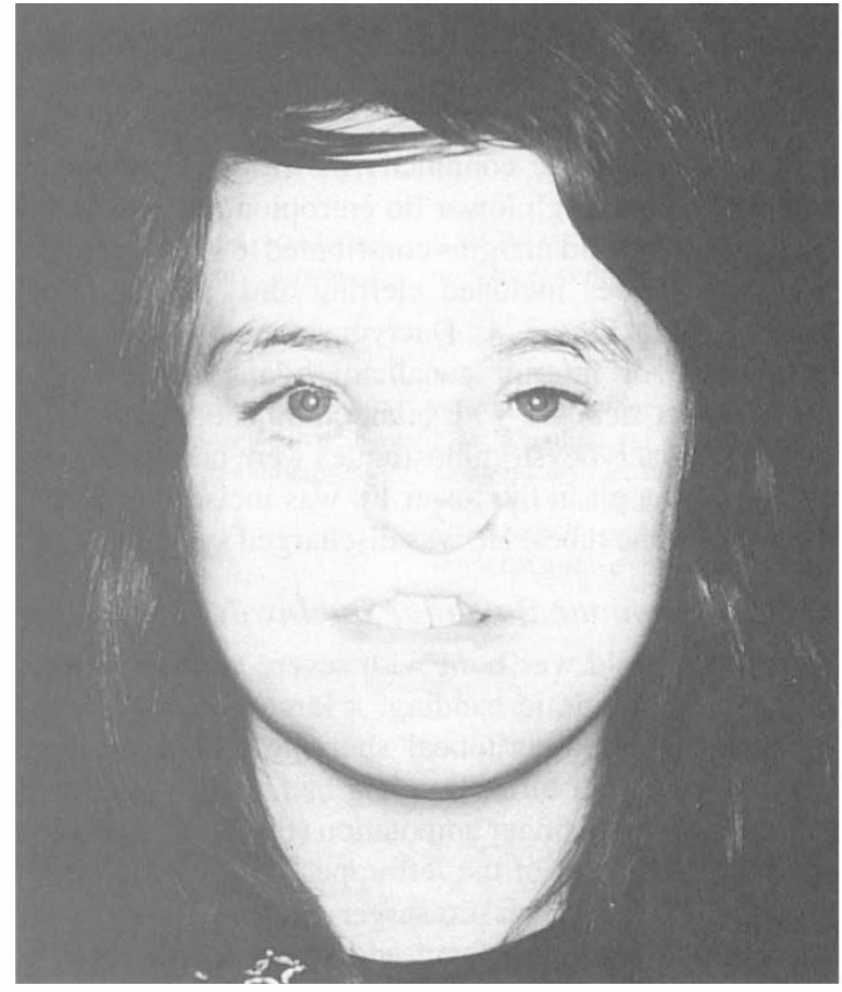

Fig. 9. Case 16. Final outcome. 


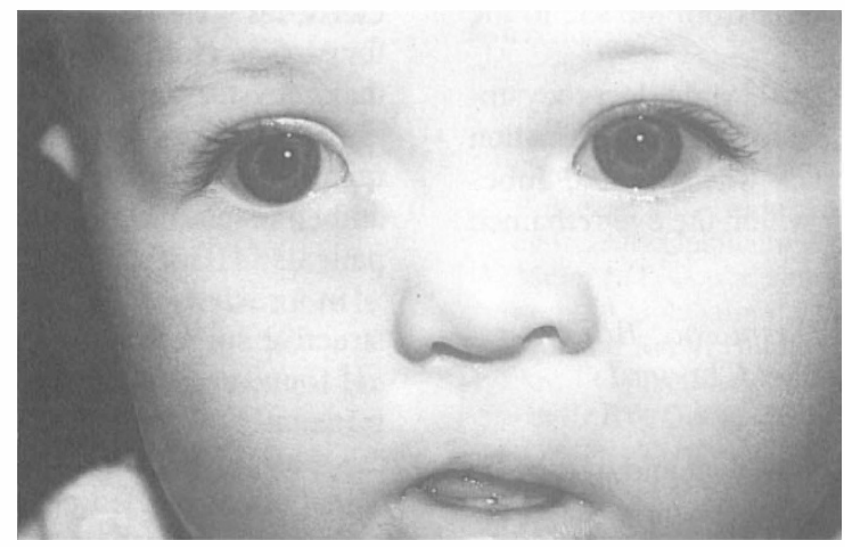

Fig. 10. Case 14. Dacryocystitis with fistula.
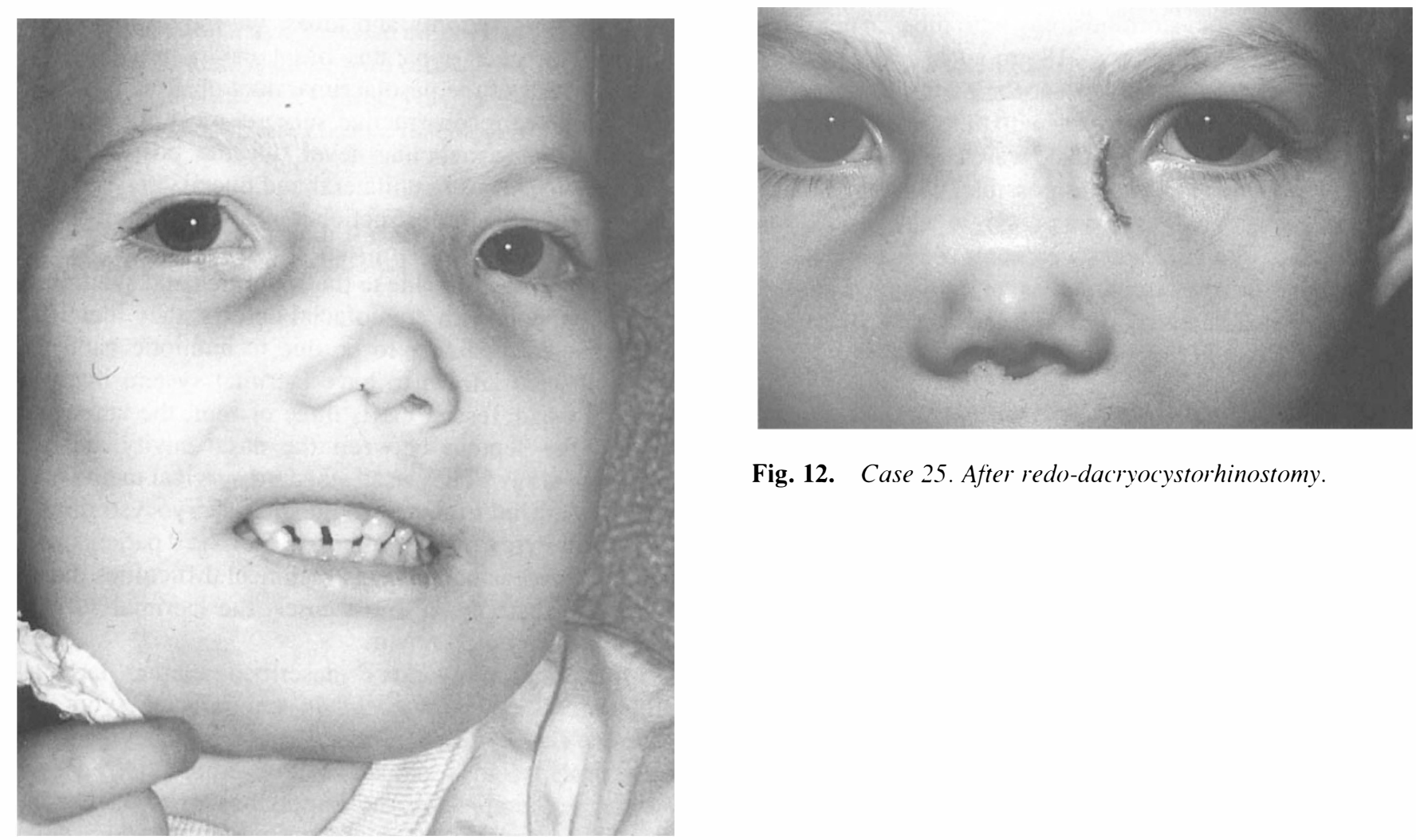

Fig. 12. Case 25. After redo-dacryocystorhinostomy.

Fig. 11. Case 25. Craniometaphyseal dysplasia.

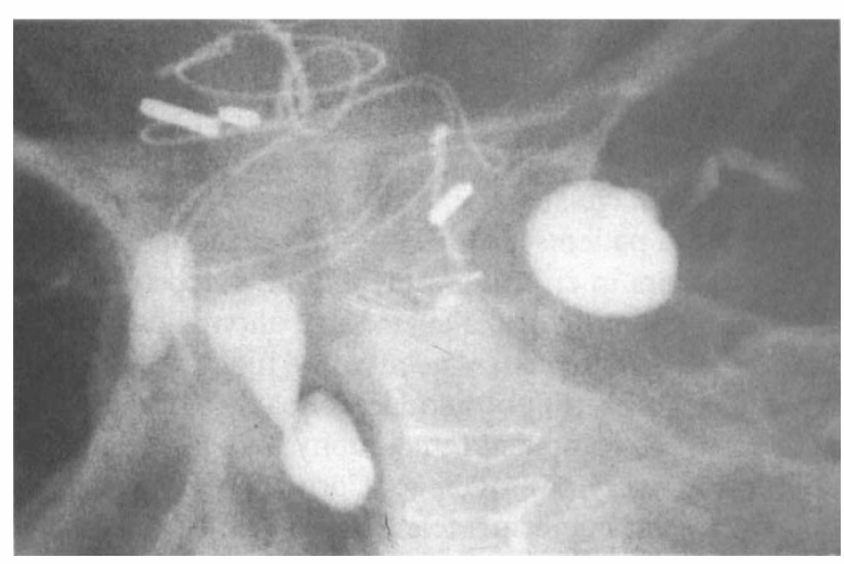

Fig. 13. Case 23. Dacryocystogram showing left mucocoele.

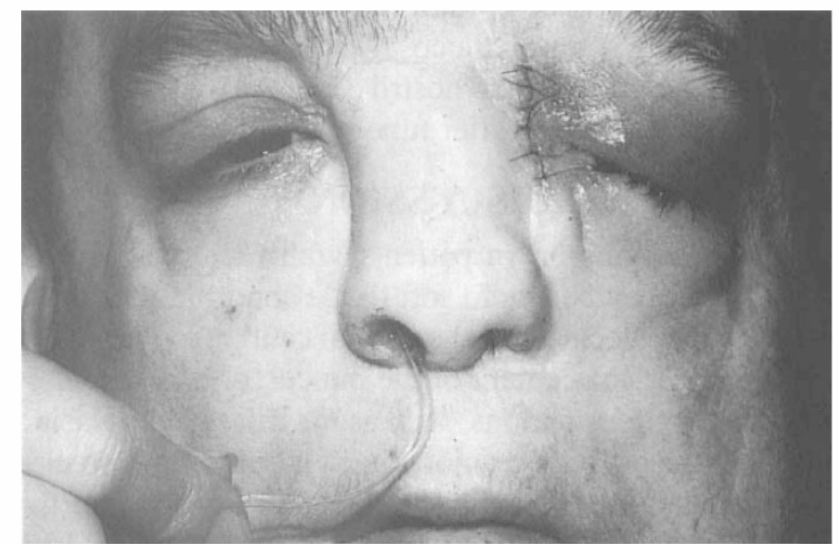

Fig. 14. Case 23. After left dacryocystorhinostomy; tubes pas sing through right nostril. 
weeks, developed an acquired fistula from the sac to the skin (Fig. 10).

At the age of 1 year he underwent a right dacryocystorhinostomy and tubes were placed due to the inflammation of the sac mucosa. The fistula track was curetted. Tubes were removed 8 weeks later after which the eye remained dry and the fistula closed.

\section{Case 25: Craniometaphyseal Dysplasia; Bony Encroachment upon the Duct and Choanal Stenosis}

Bony overgrowth of the nasolacrimal duct and dysplastic bone encroaching upon the nasal airway caused left epiphora in this female child. Other problems included a left facial palsy due to bony encroachment on the facial canal, a broad nasal bridge and a large jaw (Fig. 11). She underwent a left dacryocystorhinostomy with tubes at her referring hospital when aged 18 months, followed by recurrence of epiphora and mucocoele after initial relief. A redo-dacryocystorhinostomy with tubes was performed aged 3 years: bone $2 \mathrm{~cm}$ thick was found and the rhinostomy was made as large as possible, with all the nasal mucosa being used to form a single anastomosis of anterior flap (Fig. 12). Tubes were removed 6 months later and the patient was discharged asymptomatic. Histology of the bone specimens showed unusually prominent osteoblastic activity and limited resorption.

\section{Case 23: Midline Anomaly; Absent Internal and External Nares}

A 36-year-old man had been born with a very severe midline facial anomaly and had required extensive craniofacial reconstruction in childhood. In particular, a nose was constructed out of cartilage and skin grafts, but there was no nasal airway on the left side. On the right side the nasolacrimal system was functional. He had had a left mucocoele for 3 years, which had persisted in spite of a partial sac excision (Fig. 13). Other problems included a decompensated vascularised left cornea, hypertelorism and mild mental retardation.

Dacryocystography confirmed the absence of a nasal airway on the left and, at dacryocystorhinostomy, a $2 \mathrm{~cm}$ diameter left mucocoele was anastomosed across the midline to the right nasal mucosa and O'Donoghue tubes passed through the right nostril (Fig. 14). He was discharged symptom-free after tube removal.

\section{DISCUSSION}

Epiphora is common in patients with craniofacial syndromes and may be due to soft tissue abnormalities, such as lateral displacement of the medial canthi (primary telecanthus), or displacement of the puncta, or due to underlying bony malformations, such as maxillary hypoplasia. ${ }^{3}$

Due to the rarity of craniofacial syndromes, the precise causes of lacrimal obstruction in these patients and the surgical prognoses have not been clearly defined. In a survey of 142 children reviewed by Welham and Hughes, ${ }^{4}$ only 8 had craniofacial defect, 7 with palatal or facial clefts; all were functionally cured by dacryocystorhinostomy. One child with hemifacial microsomia underwent dacryocystectomy.

In a review of 49 patients with mild facial microsomias, regarded as insufficient for syndrome diagnosis, Hertle and colleagues ${ }^{5}$ found lid or adnexal abnormalities in 20 patients (41\%), and 7 (14\%) with dacryostenosis.

In a review of 170 patients having craniofacial reconstructive surgery for congenital deformities, Whitaker et al. ${ }^{6}$ found nasolacrimal problems in $13 \%$; these were most frequently associated with orbital hypertelorism or hypotelorism (29\%), or with craniofacial dysostosis (17\%). They recommended correction of the nasolacrimal duct problems as a secondary procedure, best performed 12 or more months after initial bone reconstructive surgery. Most of the patients in their series were treated with dacryocystorhinostomy and tubes, via the normal punctum-nasolacrimal apparatus-nasal cavity route. Sixtythree per cent of the nasolacrimal duct obstructions were present before reconstructive surgery, most occurring at the ductal or canalicular level. Of the post-operative obstructions, all were unilateral and ductal.

Lacrimal outflow obstruction may also occur secondarily to facial clefting: Hollsten and Katowitz $\mathrm{z}^{7}$ looked at patients with clefting due to the amniotic band syndrome. Of 569 patients with craniofacial defects, they identified 14 with clefting likely to be due to amniotic bands of whom 9 were known to have lacrimal system involvement. Six had Tessier clefts three or four, the latter preserving the septum between the nasal cavity and the maxillary sinus. ${ }^{8}$ They used standard surgical methods (2 dacryocystorhinostomy, 3 conjunctivodacryocystorhinostomy with Pyrex bypass tubing) in 5 of the 9 patients with lacrimal involvement, noting technical difficulties due to abnormal anatomy; in most cases, the lacrimal surgery was done after cleft repair.

Jordan and co-workers ${ }^{9}$ described another lacrimal problem which may occur in craniofacial syndrome patients: that of lacrimal gland prolapse. They describe such prolapse in a patient with Saethre-Chotzen syndrome, and emphasise the importance of replacing the gland for normal function and cosmesis.

Lauritzen and Lilja ${ }^{10}$ described standard surgical techniques for nasolacrimal surgery in patients with clefts, but did not discuss surgical problems specific to craniofacial patients, or surgical outcome.

Our series of patients with lacrimal problems associated with congenital cranial or facial anomalies shows that standard drainage surgical techniques can be adapted for use on these patients with a very high success rate.

Key factors in ensuring a favourable outcome include thorough definition of the anatomical anomalies pre- and per-operatively, with assessment of lid function and punctae and identification of fistulae; an ear, nose and throat assessment or computed tomography may be required in addition to dacryocystography. Correcting associated lid defects, to restore normal tear drainage function as far as possible, is often better done prior to dacryocystorhinostomy and in some cases is curative alone. 
Even in the presence of abnormally thick bone (cases 2, $12,22,25$ and 26) the creation of a large bone ostium and the creation of an anastomosis to whatever mucosal-lined space is available, crossing the midline if necessary (case 23), is also crucial for success; this can be done without increasing the size of the skin incision. Intubation is advisable whenever the anatomy is abnormal or there is current inflammation. Primary placement of Lester-Jones tubes is rarely, if ever, required.

When planning surgery, the expected benefits must be weighed against the possible anaesthetic risks in these patients who frequently have airway abnormalities. Timing of surgery is also important and, in general, major craniofacial reconstruction takes priority, but lacrimal surgery should be done early in patients with a large mucocoele, dacryocystitis or discharging fistulae; the source of infection may otherwise impair facial healing. In some patients, such as those with ectodermal dysplasia, it may be advisable to defer surgery as long-term dry eye problems may supersede chronic discharge.

We gratefully acknowledge Mr Richard Welham, under whose care many of these patients were treated.
Key words: Congenital, Cranial anomalies, Facial anomalies, Lacrimal dysfunction.

\section{REFERENCES}

1. Langman J. Medical embryology. 6th ed. Baltimore: Williams \& Wilkins, 1976.

2. Busse $\mathrm{H}$. Involvement of the lacrimal passages in facial clefts. Adv Ophthalmol Plast Reconstr Surg 1984;3:135-44.

3. Miller MT. Ocular abnormalities in craniofacial malformation. Int Ophthalmol Clin 1984;24:143-63.

4. Welham RAN, Hughes SM. Lacrimal surgery in children. Am J Ophthalmol 1985;99:27-34.

5. Hertle RW, Quinn GE, Katowitz JJ. Ocular and adnexal findings in patients with facial microsomias. Ophthalmology 1992;99:114-9.

6. Whitaker LA, Katowitz JA, Jacobs WE. Ocular adnexal problems in craniofacial deformities. J Max-fac Surg 1979;7:55-60.

7. Hollsten DA, Katowitz JA. The ophthalmic manifestations and treatment of the amniotic band syndrome. Ophthalmic Plast Reconstr Surg 1990;6:1-15.

8. Stretch JR, Poole MD. Nasolacrimal abnormalities in oblique facial clefts. Br J Plast Surg 1990;43:463-7.

9. Jordan DR, Germer BA, Anderson RL, Morales L. Lacrimal gland prolapse in craniosynostosis syndromes and poor function congenital ptosis. Ophthalmic Surg 1990; 21:97-101.

10. Lauritzen C, Lilja J. Nasolacrimal obstruction in craniofacial surgery. Scand J Plast Reconstr Surg 1985; 19:269-72. 